\title{
Peptidome Analysis of Pancreatic Tissue Derived from T1DM Mice: Insights into the Pathogenesis and Clinical Treatments of T1DM
}

\author{
Fan Zhang $\mathbb{D}^{1,2}$ Meiyun Zhou $\mathbb{D}^{1},{ }^{1}$ Shuangshuang Li $\mathbb{D},,^{1}$ Jinhua Gu $\mathbb{D}^{3}{ }^{3}$ Yuanyuan Qian $\mathbb{D}{ }^{1}$ \\ Sisi He $\mathbb{D}^{1},{ }^{1}$ Li Hong $\mathbb{D}^{1},{ }^{1}$ Linlin Sun $\mathbb{D}^{3},{ }^{3}$ Xiaohua Zhang $\mathbb{D}^{1}$, and Weigang Ji ${ }^{1}$ \\ ${ }^{1}$ Department of Pediatrics, Affiliated Maternity and Child Health Care Hospital of Nantong University, Nantong, Jiangsu, China \\ ${ }^{2}$ Department of Nantong Institute of Genetics and Reproductive Medicine, Affiliated Maternity and Child Health Care Hospital of \\ Nantong University, Nantong, Jiangsu, China \\ ${ }^{3}$ Department of Clinical Pharmacy, Affiliated Maternity and Child Health Care Hospital of Nantong University, Nantong, \\ Jiangsu, China
}

Correspondence should be addressed to Xiaohua Zhang; hz981218@163.com and Weigang Ji; 13606294569@163.com

Received 29 March 2021; Accepted 14 May 2021; Published 22 May 2021

Academic Editor: Cem Karadeniz

Copyright (c) 2021 Fan Zhang et al. This is an open access article distributed under the Creative Commons Attribution License, which permits unrestricted use, distribution, and reproduction in any medium, provided the original work is properly cited.

Bioactive peptides attract growing concerns for their participation in multiple biological processes. Their roles in the pathogenesis of type 1 diabetes mellitus remain poorly understood. In this study, we used LC-MS/MS technology to compare the peptide profiling between pancreatic tissue of T1DM mice and pancreatic tissue of matched control groups. A total of 106 peptides were differentially expressed in T1DM pancreatic tissue, including 43 upregulated and 63 downregulated peptides. Most of the precursor proteins are insulin. Further bioinformatics analysis (GO and pathway analysis) indicated that the potential functions of these differential peptides were tightly related to regulation of endoplasmic reticulum stress. In conclusion, this study highlights new candidate peptides and provides a new perspective for exploring T1DM pathogenesis and clinical treatments.

\section{Introduction}

Type 1 diabetes mellitus (T1DM) is a common autoimmune disease characterized by the apoptosis of $\beta$ cells and absolute deficiency of insulin secretion, which leads to chronic hyperglycemia, polydipsia, ketoacidosis, and other metabolic disorder $[1,2]$. In recent years, the global incidence of T1DM has increased rapidly. It is estimated to account for $5-10 \%$ of all diabetes cases worldwide and is expected to surge by $3 \%$ per year [3]. The etiology and pathogenesis of T1DM are complex and have not been fully elucidated up to now. At present, the main clinical treatment is insulin injection, while insulin dose, pain of injection, anxiety, and social acceptability bringing great physical and mental trauma to the patients [4]. Therefore, it is urgent to further understand the pathogenesis of T1DM and explore effective treatment approaches.

Endogenous peptides are bioactive substances that can regulate many physiological and pathological processes, including anti-infection, metabolic regulation, antioxidant, and immune regulation $[5,6]$. In recent years, a variety of bioactive peptides have been successfully isolated and identified from human, animal, and plant. For instance, cathelicidin is an antimicrobial peptide that not only protects against islet inflammation but also alleviates autoimmune diseases $[7,8]$. Amylin, a bioactive peptide, is present in pancreas cell and plays a crucial role in regulating blood sugar [9]. In addition, glucagon-like peptide 1 (GLP-1) receptor agonists such as Lilarutin have been used as drugs for the treatment of diabetes [10]. These studies stimulated our 


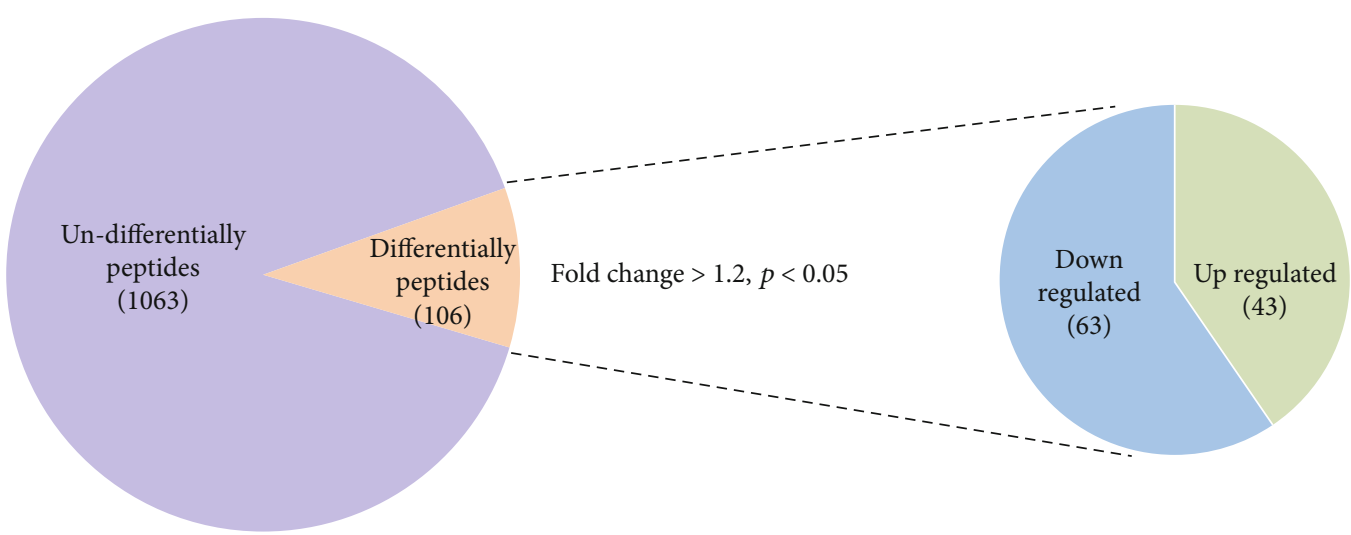

(a)

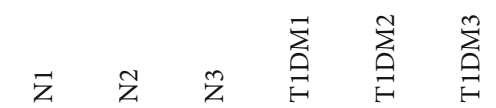

Rplp2_SAAPGSAAPAAGSAP

Krt8_GMSSFQPGFGSAGGSN

Hnrnpa2b1_GSDGYGSGRGFGDG

Actb_DIAALVVDN

Rpsa_APTAQATEWVGATTE

Gm45713_TEVLKTNGLLV

N/A_VTLASHHPADFTPAVH

Hnrnpa2b1_SDGYGSGRGFGDG

Gm45713_TEVLKTNGLL

Hsp90b1_SLTDENALAGNEELT

Krt8_GLGGFGGAGVGGIT

Rpl31_KNLQTVNVDEN

Krt8_FQPGFGSAGGSN

Tpd52_MDRGEQGLLKTEPV

Hnrnpa2b1_GGSDGYGSGRGFGDG

Tubala_DLEPTVIDEVRTGT

Sec61a1_ELGISPIVTSG

Rbm3_DYSGRSQGGYD

Cct7_GVDINNENIADN

SARNP_FGIVTSSAGTGTTEDTEA

$\begin{array}{llllllll}14 & 12 & 10 & 8 & 6 & 4 & 2 & 0\end{array}$

(b)

Figure 1: Continued. 


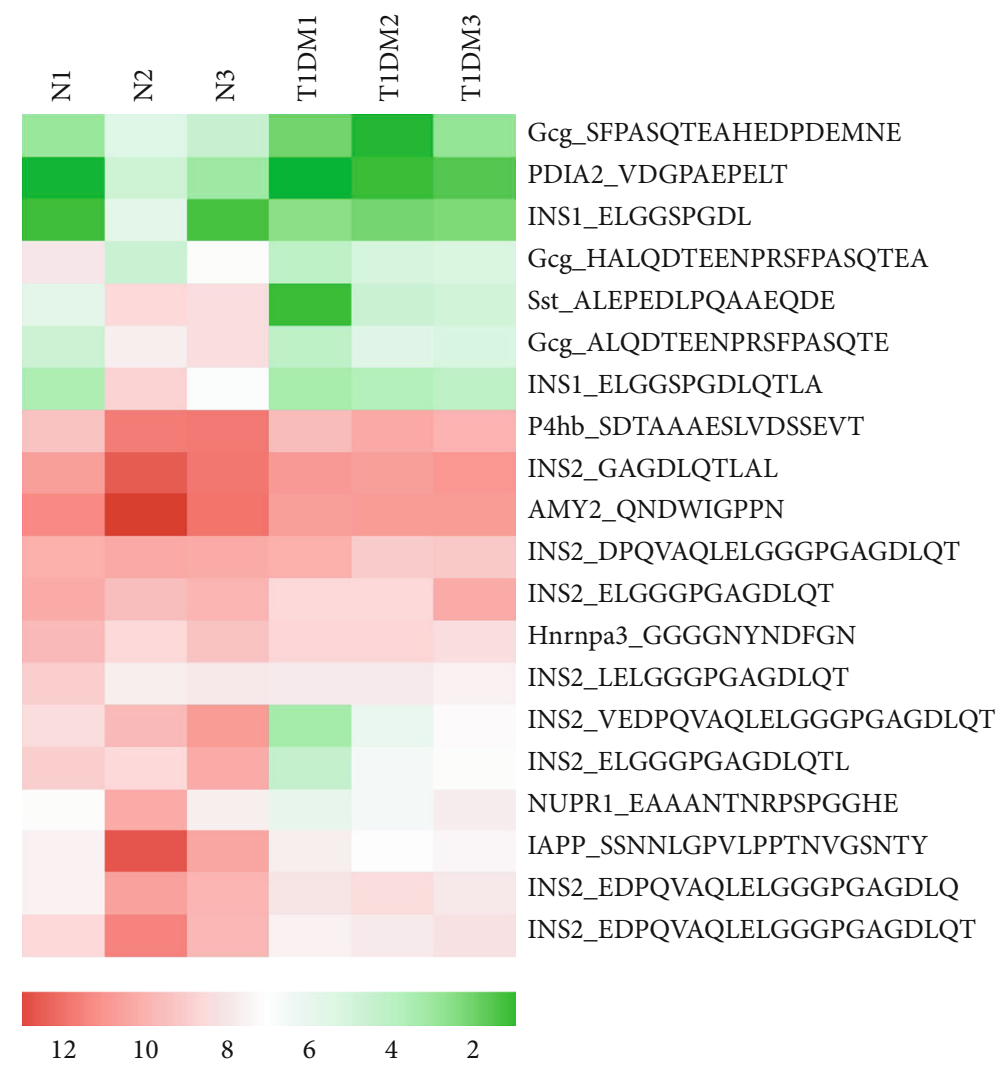

(c)

Figure 1: Differently expressed peptides between T1DM and normal mice. (a) Of the 1,063 nonredundant peptides, 106 peptides were significantly differentially expressed in the T1DM mice (fold change $\geq 1.2$ and $p<0.05$ ), including 43 upregulated and 63 downregulated peptides. Hierarchical clustering showed significant differences in expression between the two groups. The twenty upregulated (b) and downregulated (c) peptides with the highest fold changes were displayed. $N$ : control; T1DM: type 1 diabetes mellitus; high expression: red; low expression: green.

interest in the study of bioactive peptides associated with diabetes. However, the function of the bioactive peptides in T1DM pathogenesis has rarely been researched.

Herein, liquid chromatography tandem mass spectrometry (LC-MS/MS) was employed to compare bioactive peptides in different pancreatic tissue from control and T1DM model mice. The bioinformatics characteristics and cleavage sites of these differentially expressed peptides were also analyzed. Furthermore, we characterized these differentially expressed peptides using Gene Ontology (GO) and pathway analyses and explored potential peptides associated with T1DM. The data suggested that the altered peptidomic profiles in the pancreatic tissues may play an important role in T1DM development.

\section{Methods}

2.1. Animal Model. Wild-type C57BL/6J mice (male, 6 weeks old) were purchased from the Experimental Animal Center of Nantong University. All mice were housed in a specificpathogen free room with a $12 \mathrm{~h}$ light-dark cycle and adequate chow and water. The animals were randomly divided into two groups: T1DM group and control group. The mice of the T1DM group were fasted overnight and then intraperito- neally injected with STZ (Sigma-Aldrich) at a dose of $45 \mathrm{mg} / \mathrm{kg}$ body weight for 5 consecutive days, whereas the mice of control group received the same volume of citrate acid-sodium citrate buffer $(\mathrm{pH} 4.54)$. Mice with random blood glucose levels greater than $16.7 \mathrm{mM}$ were considered diabetic. This experiment was approved by the Animal Ethics Committee of the Affiliated Maternity and Child Health Care Hospital of Nantong University in accordance with the National Institutes of Health Guide for the Care and Use of Laboratory Animals.

2.2. Peptide Extraction. Each collected pancreatic tissue was washed with precooled PBS, then ground into powder in liquid nitrogen, and finally mixed with a four times volume of lysate (PBS containing $1 \mathrm{mM}$ PMSF). The mixture was placed on ice for 5 minutes and then centrifuged at 12,000 $\mathrm{g}$ for $10 \mathrm{~min}$ at $4^{\circ} \mathrm{C}$. The equal volume of $100 \% \mathrm{ACN}$ (acetonitrile) was added to the supernatant and then centrifuged at $10,000 \mathrm{~g}$ for $10 \mathrm{~min}$. Transfer the freeze-dried supernatant to a prewetted $10 \mathrm{kD}$ ultrafiltration tube (Merck Millipore, UFC501096, Germany) and centrifuged at 10,000 $\mathrm{g}$ for $20 \mathrm{~min}$ at $4^{\circ} \mathrm{C}$. The filtrates were collected, and the peptides were desalted using C18 columns. The desalted peptide 


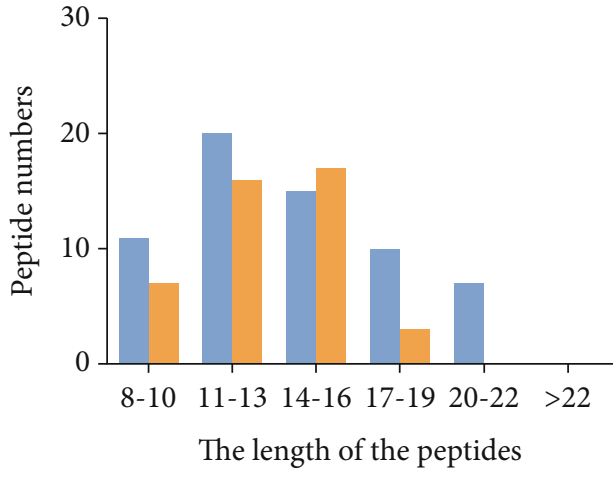

Down-regulated

Up-regulated

(a)

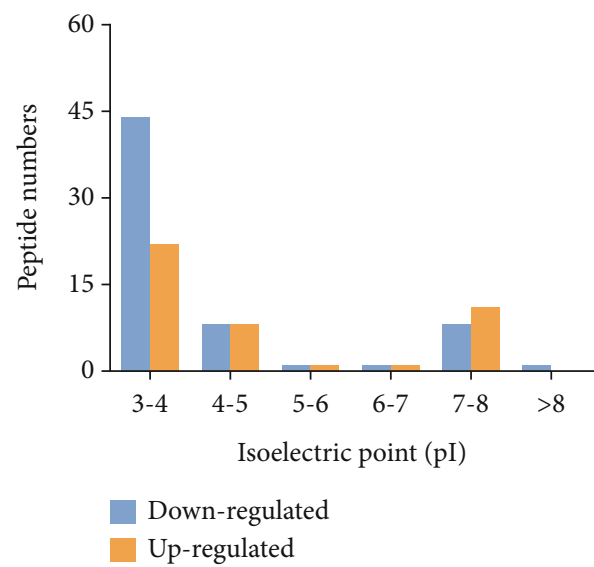

(c)

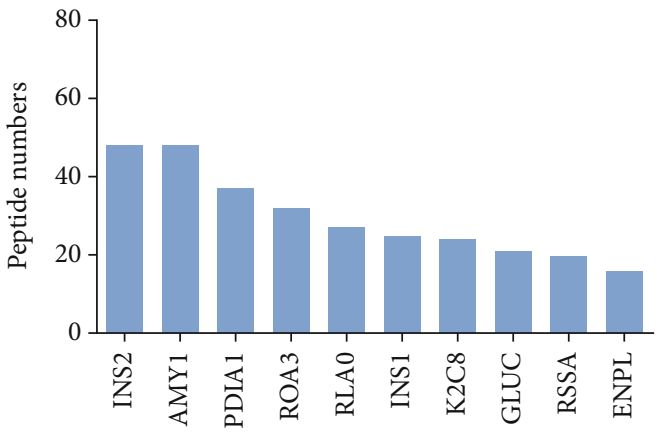

Names of precursor proteins

(e)

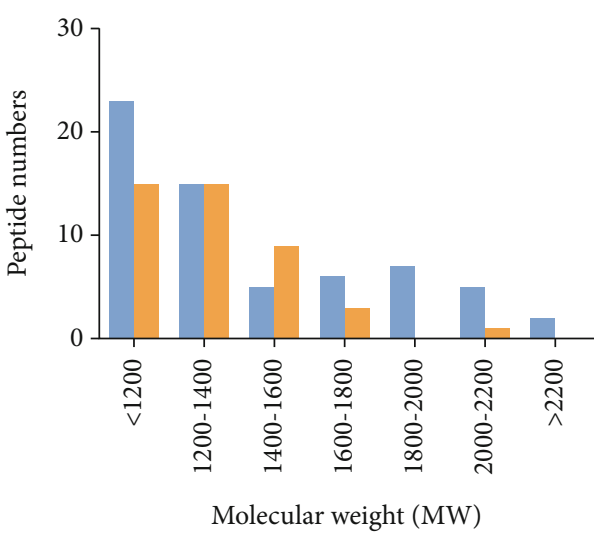

Down-regulated

Up-regulated

(b)

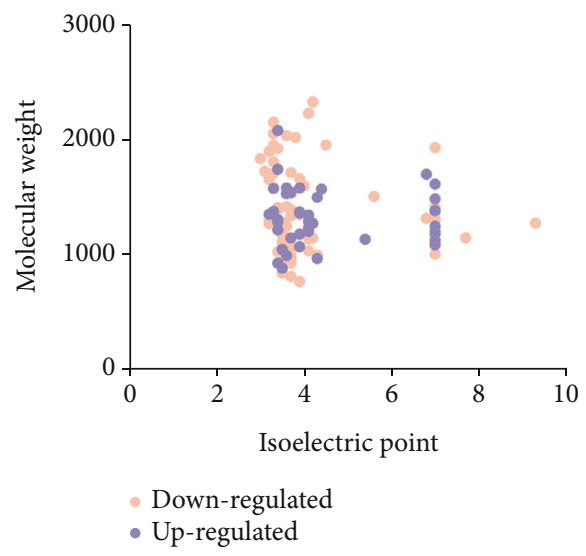

(d)

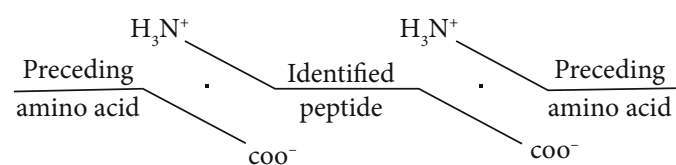




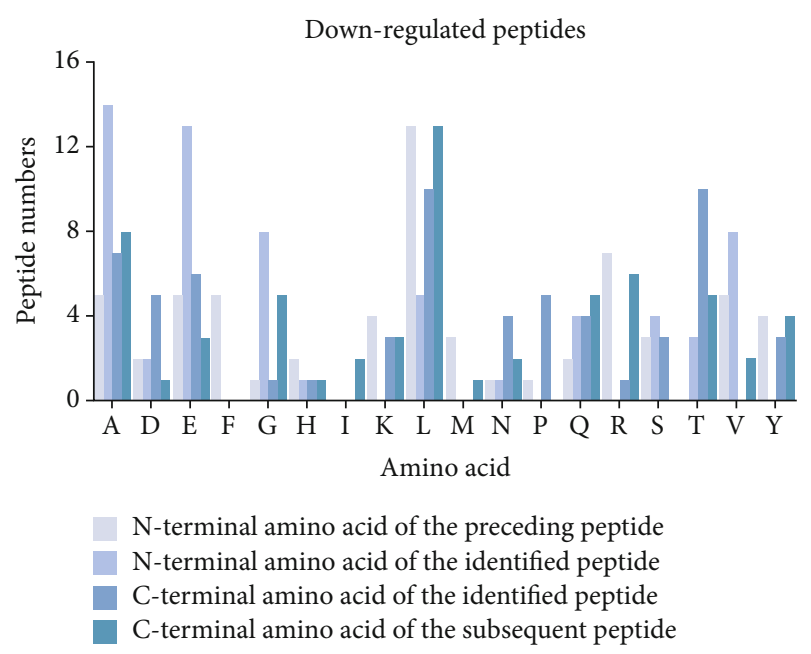

(g)

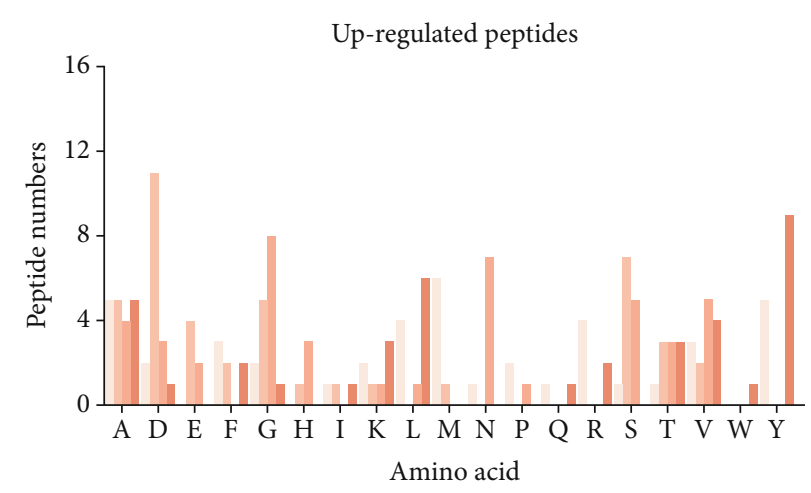

N-terminal amino acid of the preceding peptide $\mathrm{N}$-terminal amino acid of the identified peptide

C-terminal amino acid of the identified peptide

C-terminal amino acid of the subsequent peptide

(h)

Figure 2: Characteristics of differentially expressed peptides: (a) amino acid of peptides; (b) molecular weight (MW); (c) isoelectric point (pI); (d) scatter plot of MW versus pI; (e) peptides shared the same precursor proteins; (f) cleavage method of peptide; (g) four cleavage sites in downregulated peptides; (h) four cleavage sites in upregulated peptides.

solution was vacuum dried and immediately frozen at $-80^{\circ} \mathrm{C}$ until MS analysis.

\subsection{Isobaric Tags for Relative and Absolute Quantitation} (iTRAQ). The peptides were dissolved with $0.5 \mathrm{M}$ TEAB $(100 \mu \mathrm{g})$, labeled according to manufacturer's instructions of iTRAQ-8 kit (SCIEX), and then, the mixed peptide samples were separated by grade using Ultimate 3000 HPLC system (Thermo DINOEX, USA). The chromatographic column was Durashell C18 column $(5 \mathrm{~m}, 100 \mathrm{~A}, 4.6 \times 250$ $\mathrm{mm})$. The concentration of ACN gradually increased under alkaline conditions to separate the peptide segment, and the flow rate was $1 \mathrm{ml} / \mathrm{min}$, and one tube was collected every minute. A total of 42 secondary fractions were collected and incorporated into 12 components, which were desalted and vacuum dried on the strata-x column.

2.4. LC-MS/MS Analysis. The peptide samples were redissolved in a $2 \%$ acetonitrile/0.1\% formic acid (FA) solution and analyzed using a TripleTOF 5,600 plus mass spectrometer coupled to an Eksigent NanoLC System (SCIEX, USA). Peptides were loaded onto a C18 trap column $(5 \mu \mathrm{m}, 100 \mu$ $\mathrm{m} \times 20 \mathrm{~mm}$ ) and eluted at $300 \mathrm{nl} / \mathrm{min}$ onto a C18 analytical column $(3 \mu \mathrm{m}, 75 \mu \mathrm{m} \times 150 \mathrm{~mm})$ in the gradient as long as $90 \mathrm{~min}$. These two mobile phases included buffer A ( $2 \%$ acetonitrile/0.1\% formic acid/98\% $\mathrm{H}_{2} \mathrm{O}$ ) and buffer B ( $98 \%$ acetonitrile $/ 0.1 \%$ formic acid/2\% $\mathrm{H}_{2} \mathrm{O}$ ). For informationdependent acquisition (IDA), the first-order mass spectrogram was scanned at $250 \mathrm{~ms}$ accumulation time, and the second-order mass spectrogram of 30 precursor ions was collected at $50 \mathrm{~ms}$ accumulation time. The collections of MS1 spectra were in the range $350-1500 \mathrm{~m} / \mathrm{z}$, and MS2 spectra were in the range of $100-1500 \mathrm{~m} / \mathrm{z}$. The dynamic exclusion time of the precursor ions was set to $15 \mathrm{~s}$.

2.5. MS/MS File Data Analysis. ProteinpilotTM v4.5 was adopted to analyze the original MS/MS file data. For peptides identification, the Paragon algorithm was employed against the Mus-musculus SwissProt sequence database. We further filtered these data according to the result of proteinpilot. We consider unused score $\geq 1.3$ (confidence $>95 \%$ ) and proteins containing at least one unique peptide per protein as trusted proteins. For the identified peptide and protein quantification, we consider that the credible peptide is used for protein quantification with the credibility of more than $95 \%$.

2.6. Bioinformatics Analyses. We analyzed the molecular weight (MW) and isoelectric point (pI) of the peptides by using the online tool ProtParam (http://web.expasy.org/ protparam/). The UniProt database (https://www.uniprot .org/peptidesearch/), Pfam (http://pfam.xfam.org/), and STRING (http://string-db.org/) were used to determine the potential function and interaction of peptides and their protein precursors. Gene Ontology (GO) analysis (http://www .geneontology.org), containing cellular component, molecular functions, and biological processes, was used predict the latent functions of the identified peptides. Each peptide and their protein precursors were imported into the Ingenuity Pathway Analysis software v7.1 for pathway analysis and predicting latent biologic, biochemical, and molecular functions of the given proteins, which were incorporated into an associated network map. The online search tool (https://blast .ncbi.nlm.nih.gov/Blast.cgi) was employed for homology comparison. The PyMOL Molecular Graphics System (CA) was established to model the structures of indicated peptides.

2.7. Statistical Analysis. The results of the bioinformatics analysis were analyzed using GraphPad Prism 7, and the data were shown as the mean \pm standard deviation (SD). Peptides with a fold change larger than 1.2 or $<0.83$ with a Student's $t$ -test $p$ value $<0.05$ were selected as differently expressed peptides. 


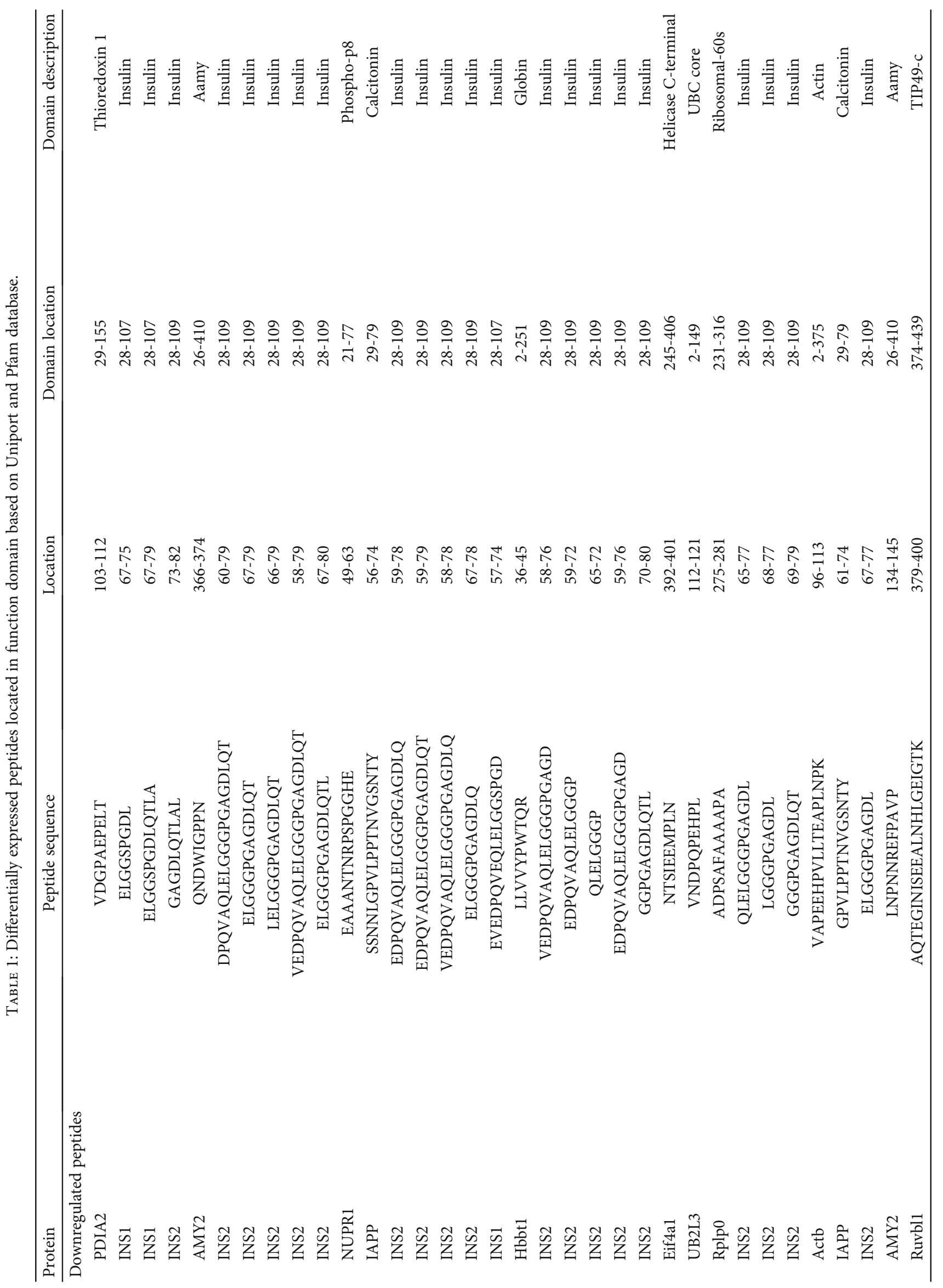




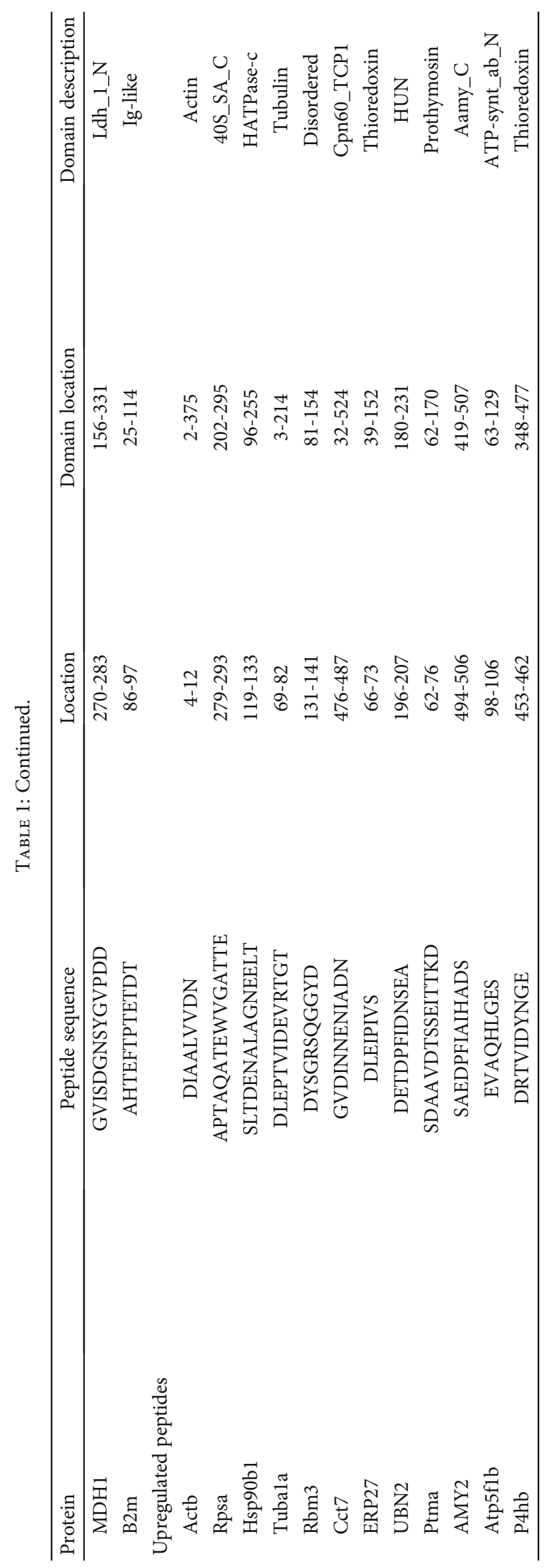


Cellular component

Cell projection membrane $\mathrm{H} 4 / \mathrm{H} 2 \mathrm{~A}$ histone acetyltransferase complex Small nucleolar ribonucleoprotein complex Histone acetyltransferase complex Pigment granule Cell projection part Melanosome

Endoplasmic reticulum lumen Leading edge membrane Cortical actin cytoskeleton

DNA helicase complex Dendrite cytoplasm

Chromatin remodeling complex

Nucleoplasm part Lysosomal lumen Cell surface Nuclear chromosome Endocytic vesicle

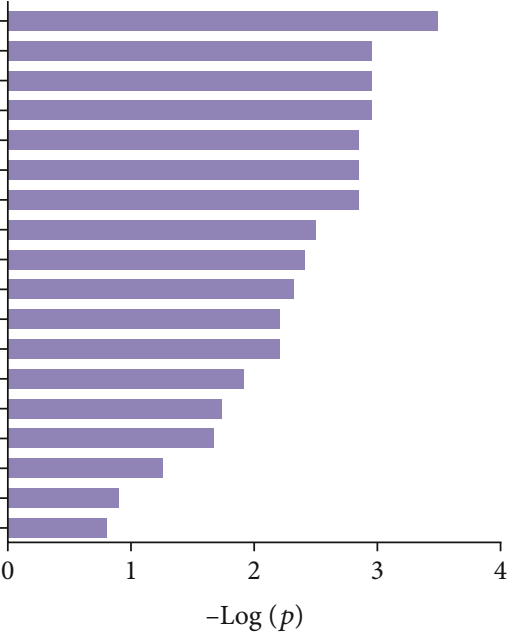

(a)

Molecular function

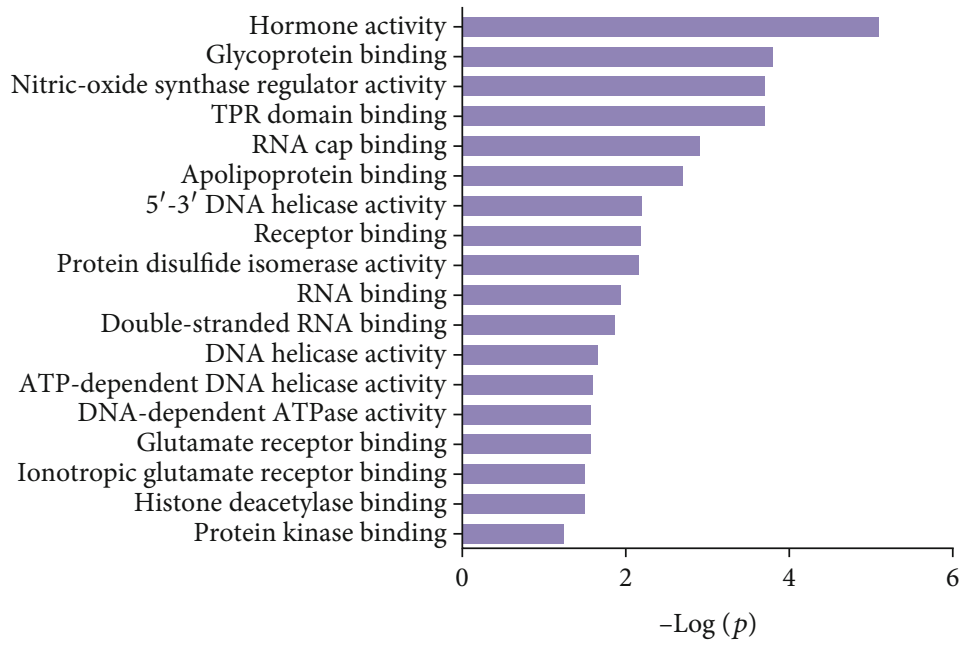

(b)

Biological process

Response to endoplasmic reticulum stress

Cellular protein metabolic process

Cellular macromolecule metabolic process Protein secretion

Wnt receptor signaling pathway
ER overload response Regulation of protein ubiquitination

Regulation of protein binding Response to osmotic stress

Regulation of lipid kinase activity

Regulation of phosphoinositide 3-kinase activity Regulation of nitric-oxide synthase activity

Cellular biopolymer metabolic process

Positive regulation of cell communication Cell surface receptor linked signal transduction

Regulation of lipid metabolic process

Protein folding

Protein targeting to mitochondrion

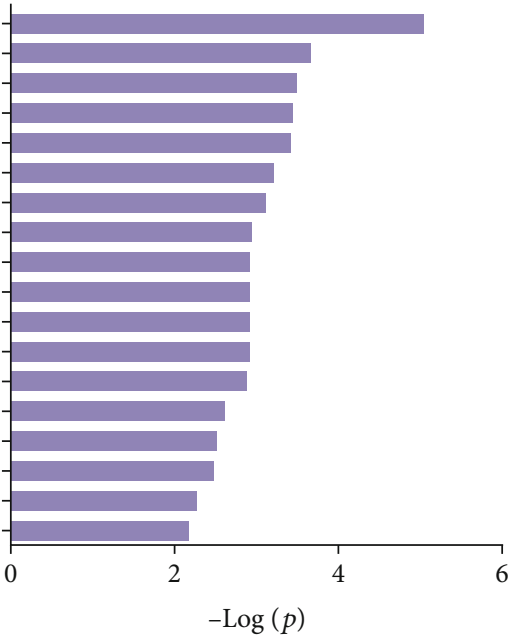

(c)

Figure 3: Continued. 


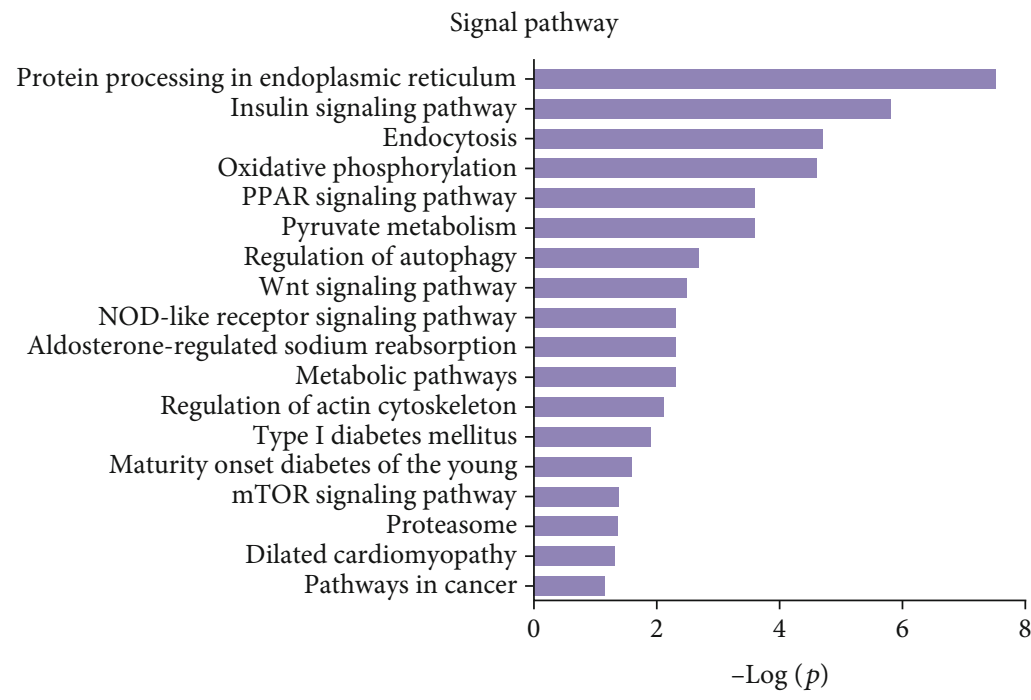

(d)

FIGURE 3: Gene Ontology (GO) and pathway analysis of these precursor proteins: (a) cellular components; (b) molecular functions; (c) biological processes; (d) pathway analysis.

\section{Results}

3.1. Identification of Differentially Expressed Peptides in the Pancreatic Tissue from T1DM Mice. Endogenous peptides from the pancreatic tissue of T1DM mice and control groups were directly analyzed using LC-MS/MS after iTRAQ labeling. A total of 1063 nonredundant peptides were identified, among which 106 peptides were differentially expressed in the T1DM mice and control groups (fold change $>1.2, p<0.05$ ), including 43 upregulated peptides and 63 downregulated peptides (Figure 1(a)). All of the differentially expressed peptides are listed in Table S1. The twenty upregulated and downregulated peptides detected in all samples had the highest range of change, which was graphically represented by hierarchical clustering analysis (Figures 1(b) and 1(c)).

\subsection{Characteristics of Differentially Expressed Peptides. To} characterize the general feature of differentially peptides, we further analyzed the relative molecular weight (MW), isoelectric point (pI), amino numbers, and distribution of $\mathrm{pI}$ vs. MW. The results shown that the majority of these peptides were 8-16 amino acids in length (Figure 2(a)); the MW varied from $769.9 \mathrm{Da}$ to $2324.5 \mathrm{Da}$ with $75 \%$ between 1000 and $1800 \mathrm{Da}$ (Figure 2(b)), and pI varied from 3.0 to 9.3, with 77\% between 3 and 5 (Figure 2(c)). Furthermore, the distribution of pI vs. MW could divide these peptides into two groups around pI3 and pI7 (Figure 2(d)). There were several peptides derived from the same precursor protein. The top ten precursor proteins are listed in Figure 2(e), and the largest number of peptides is derived from INS2. Then, UniProt and Pfam databases were used to analyze the functional domains of these peptides. A total of 36 downregulated and 12 upregulated peptides located in the domains of their precursor proteins are listed in Table 1.
3.3. Cleavage Site Patterns of Differentially Expressed Peptides. Peptides are mainly cleaved by proteolytic enzyme, and the regulation of their level depends on the type and activity of protease. In other words, by analyzing the characteristic pattern of peptide cleavage sites, the functional differences that may be caused by the enzyme cleavage process can be found. The diagram of the cleavage site distribution is showed in Figure 2(f). Four cleavage sites (C-terminal amino acid of the preceding peptide, Cterminal amino acid of the identified peptide, N-terminal amino acid of the identified peptide, N-terminal amino acid of the identified peptide) were researched for each up- and downregulated peptide (Figures 2(g) and 2(h)). The four dominant cleavage sites in the upregulated peptides were alanine $(\mathrm{A})$, lysine $(\mathrm{K})$, threonine $(\mathrm{T})$, and glycine $(\mathrm{G})$, while in the downregulated peptides were alanine $(\mathrm{A})$, lysine $(\mathrm{K})$, lysine $(\mathrm{K})$, and proline $(\mathrm{P})$.

3.4. Gene Ontology (GO) and Pathway Analysis. Gene Ontology (GO) and pathway analysis were used to further understand the potential functions of these differential peptides and their corresponding precursor proteins. Cell projection membrane, H4/H2A histone acetyltransferase complex, small nucleolar ribonucleoprotein complex, histone acetyltransferase complex, pigment granule, cell projection part, etc., were the most highly enriched cellular components (Figure 3(a)), whereas hormone activity, glycoprotein binding, nitric-oxide synthase regulator activity, TPR domain binding, RNA cap binding, etc., were the highest enriched molecular functions (Figure 3(b)). Finally, response to endoplasmic reticulum stress, cellular protein metabolic process, cellular macromolecule metabolic process, protein secretion, Wnt receptor signaling pathway, ER overload response, etc., were the most highly enriched biological processes (Figure 3(c)). The pathway analysis revealed that the differentially expressed peptides and their corresponding 


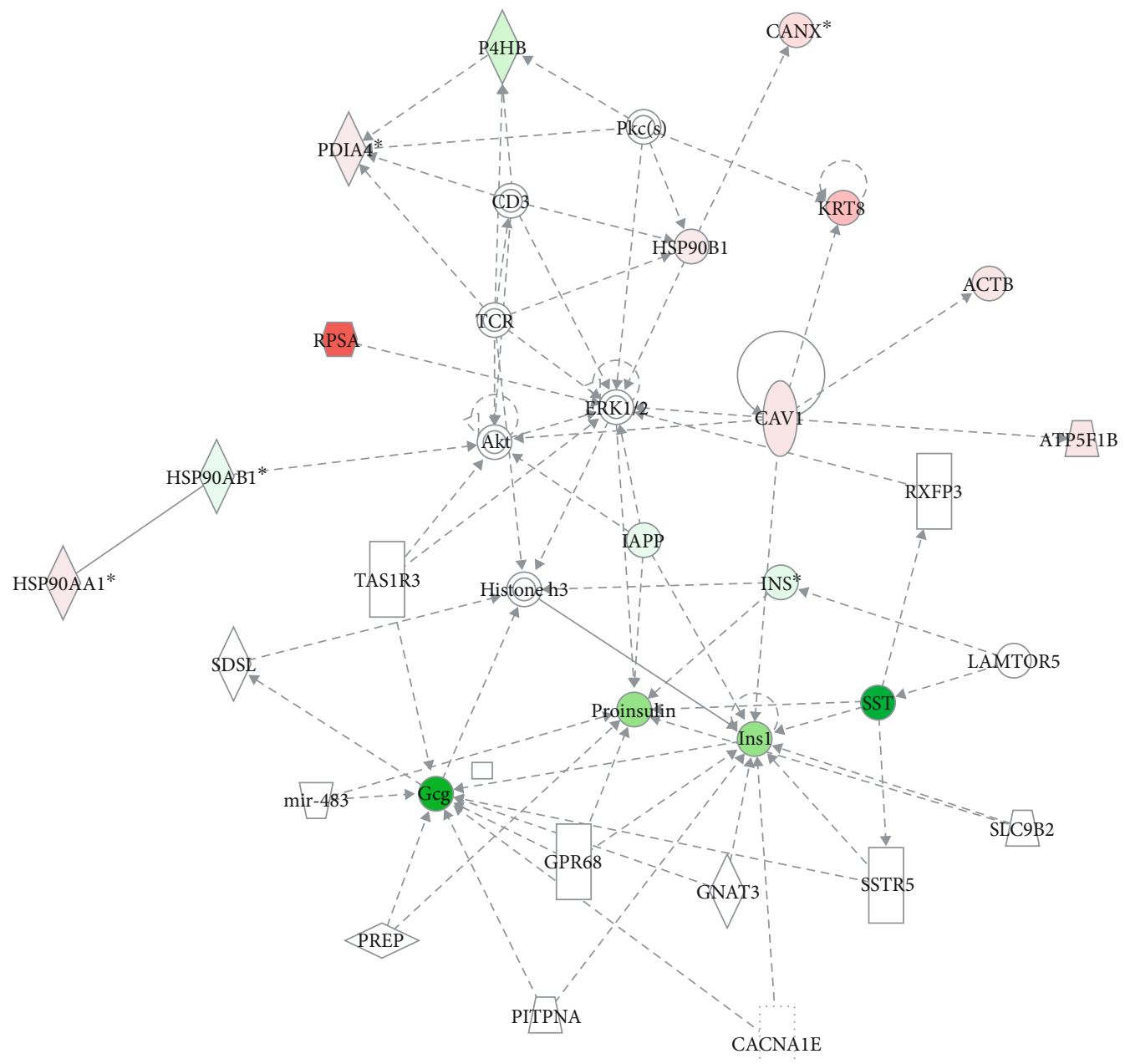

(a)

FIgUre 4: Continued. 


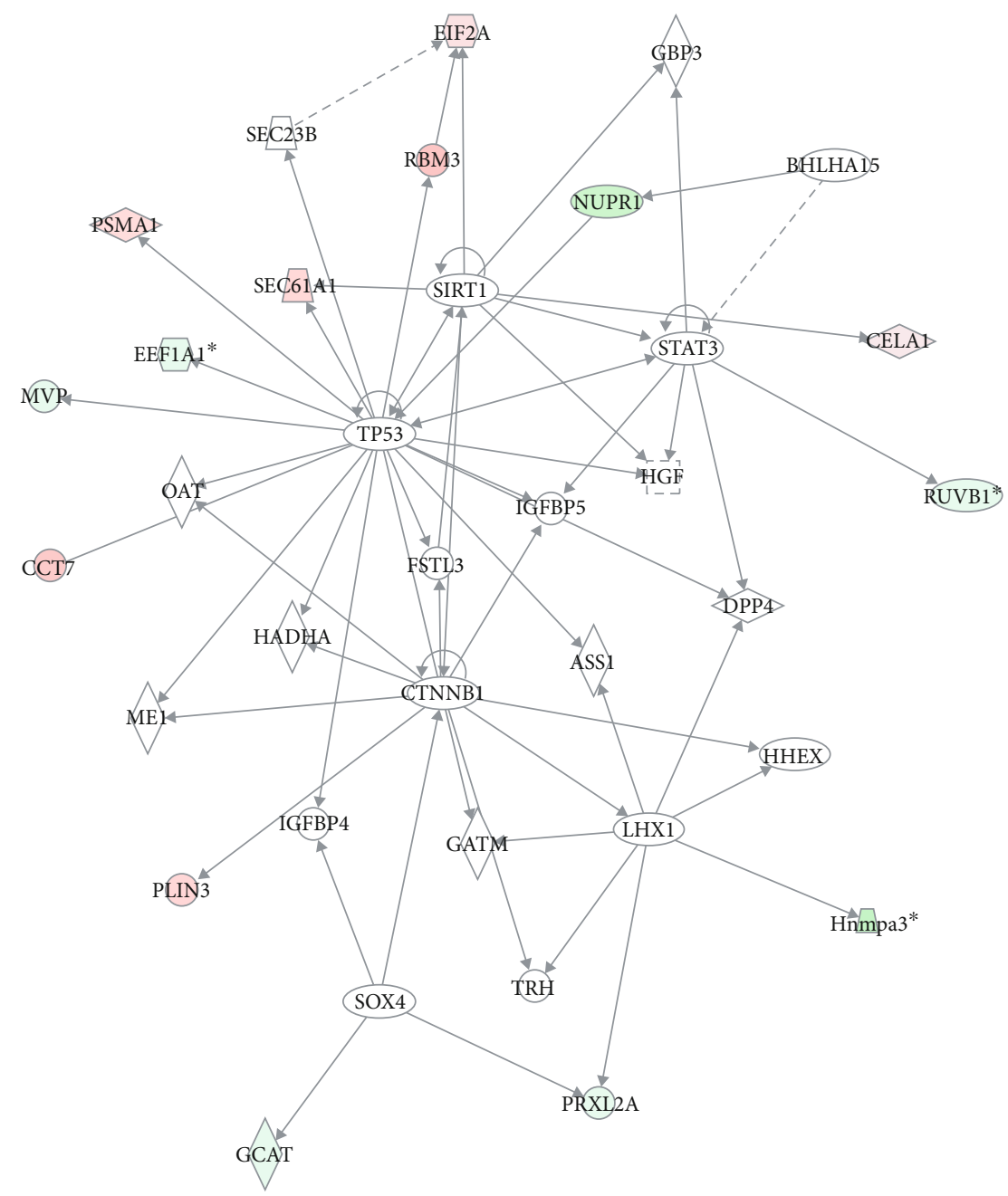

(b)

FIgure 4: Continued. 


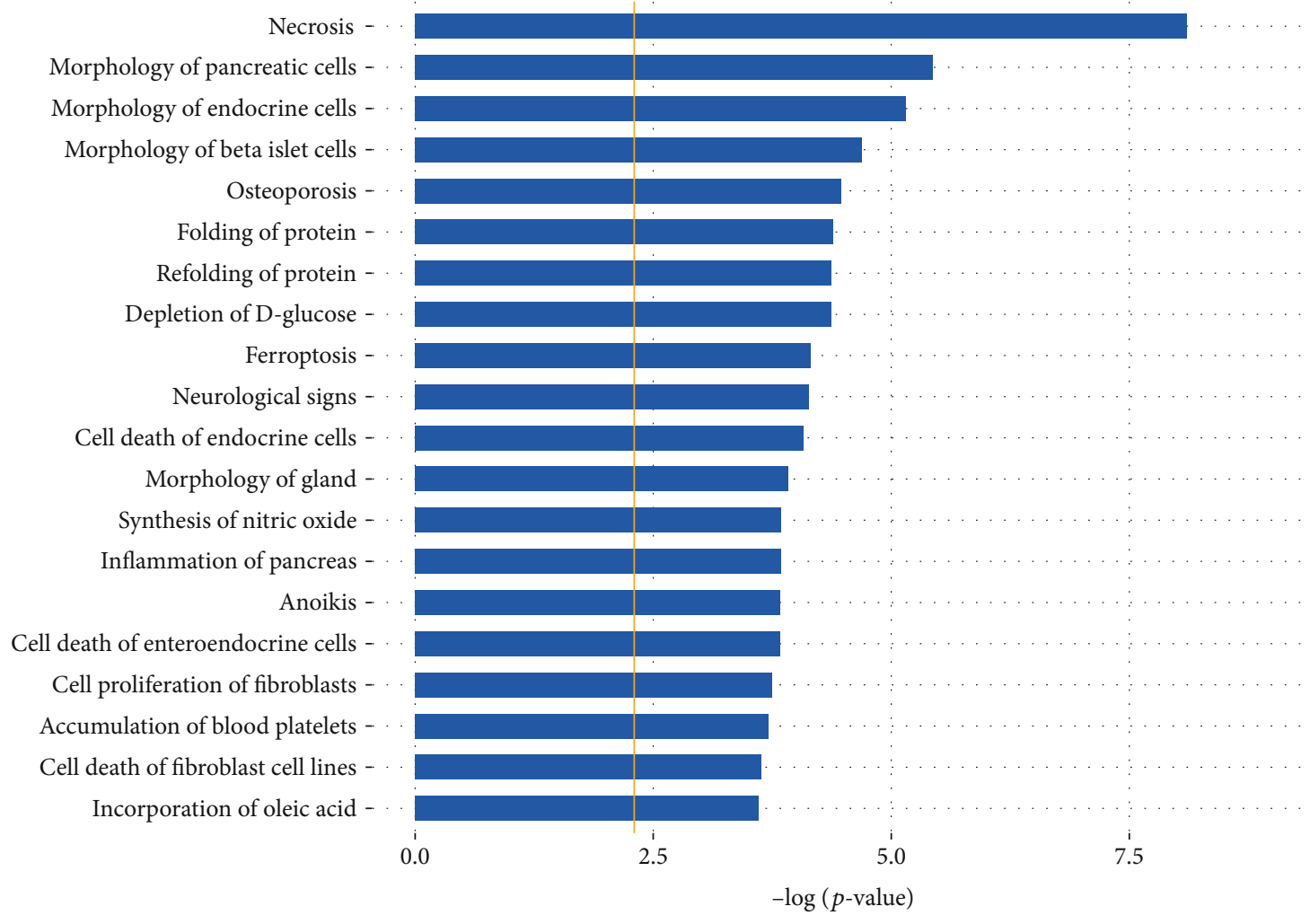

(c)

Figure 4: Functional clustering by IPA. (a) Network analysis of "cancer, endocrine system disorders, organismal injury and abnormalities". (b) Network analysis of "carbohydrate metabolism, cell death and survival, skeletal and muscular disorders" (upregulation, red; downregulation, green). (c) The top twenty precursor proteins related disease function.

precursor proteins were involved in protein processing in endoplasmic reticulum, insulin signaling pathway, endocytosis, oxidative phosphorylation, and PPAR signaling pathway, among others (Figure 3(d)).

3.5. Functional Clustering by IPA. The IPA software was employed to produce the interaction network of the precursor proteins of 106 differentially expressed peptides and high-scoring networks that may be linked to the endocrine system. Both "cancer, endocrine system disorders, organismal injury and abnormalities" and "carbohydrate metabolism, cell death and survival, skeletal and muscular disorders" were identified (Figures 4(a) and 4(b)).

The differentially expressed peptides were also entered into the IPA software to conduct a comprehensive analysis of the precursor proteins and their functional analysis of related diseases. The data analysis suggested that some of the precursor proteins were related to endocrine system development and function. These precursor proteins were predicted to have roles in necrosis $(p=7.82 E-09)$, morphology of pancreatic cells $(p=3.69 E-06)$, morphology of endocrine cells $(p=6.93 E-06)$, and morphology of beta islet cells $(p=2.02 E-05)$. The top twenty precursor proteins related disease function are listed in Figure 4(c).

\section{Discussion}

Type 1 diabetes mellitus (T1DM) is characterized by the dysfunction and apoptosis of pancreatic $\beta$ cells $[11,12]$. It occurs primarily in children and adolescents. Although T1DM is relatively rare, it still increases at an annual rate of 3\%. However, the etiology and pathogenesis of T1DM are complex and have not been fully elucidated up to now [13]. Longterm insulin injection and long-term metabolic disorders cause many acute and chronic complications. Recently, some peptide drugs have provided new ideas for the treatment of diabetes. Peptides display a multitude of biological activities such as stabilizing mitochondrial proteins, stimulating glucose uptake, and regulating signal transduction $[14,15]$. So, we attempted to synthesize the peptides that may be involved in regulating insulin secretion to provide important insights into the molecular mechanism of pancreatic $\beta$ cell apoptosis.

In our study, we collected pancreas from normal and T1DM model mice for peptide extractions with $10 \mathrm{kDa}$ MWCO filters, which could remove redundant proteins and ensure the purity of the extracted peptides. Then, we employed TMT-labeled analysis to compare the composition of pancreas peptides from the T1DM and control group and identified 1063 peptides from LC-MS/MS. A total of 106 peptides were differentially secreted in pancreas tissues of T1DM mice, of which 43 peptides were upregulated and 63 were 
downregulated. The MWs of the identified peptides were less than $2.5 \mathrm{kDa}$, and $75 \%$ of the peptides were mainly distributed in the range of $1.0-1.5 \mathrm{kDa}$. The $\mathrm{pI}$ of the peptides ranged from 3 to 5 , accounting for $77 \%$ of all peptides. This data indicated that the MWCO filter is effective in our process of extracting peptides.

Blood glucose balance regulation is a part of the body life activity regulation and is an important condition to maintain the homeostasis of the body environment. There are many kinds of hormones in human body that can regulate the content of blood glucose, but insulin and glucagon are the main ones [16-18]. In our study, 106 differentially expressed peptides from 57 precursor proteins were identified from different pancreatic tissues. Most of the precursor proteins are insulin. It is quite possible that the peptides upregulated can raise blood sugar, whereas downregulated peptides could be reduced blood sugar. Therefore, we can test the function of these peptides by gene silencing or synthetic peptide treatment experiments. Further study on the function of these differentially expressed peptides will provide a new approach for the treatment of T1DM.

Protein domain is a region with specific sequence and structure that can folding and traveling functions independently. We identified 48 peptides located in function domains, 22 of which were insulin domain. The main role of insulin is to reduce blood sugar; it can increase cell permeability to monosaccharides, amino acids, and fatty acids and is the best treatment for type 1 diabetes so far [19-21]. Thioredoxin domain is a class of small redox proteins known to be present in all organisms. Recent research shows that thioredoxin interacting protein is an important factor in regulating pancreatic $\beta$ cell dysfunction and death and is a key process in the pathogenesis of T1DM [22]. Therefore, the confirmation of these peptides is very important in the future studies.

The biological functions of these peptides were predicted by the analysis of the precursor proteins. GO and pathway analysis showed that the potential functions of these differential peptides were tightly related to regulation of endoplasmic reticulum stress. Recent observations suggest that endoplasmic reticulum stress mediated apoptosis plays an important role in pancreatic $\beta$ cell destruction $[23,24]$. The early biosynthesis of insulin is mainly in the endoplasmic reticulum, and the pancreatic $\beta$ cells have a highly active and developed ER [25]. It is one of the most sensitive cells to endoplasmic reticulum stress. Persistent ERS and defective ERS signaling pathways in $\beta$ cells can cause the dysregulation of ER homeostasis, which results in pancreatic $\beta$ cell apoptosis and autoimmune response and then developing into diabetes mellitus [26, 27]. These results indicated that these peptides participate are involved in dysfunction and death of pancreatic $\beta$ cells and play an important regulatory role.

In summary, using LC-MS/MS technology, we have identified the peptide profiles of pancreatic tissues from T1DM mice and matched normal mice. Our results suggest that some peptides may participate in pancreatic dysfunction and diabetes. The emergence of these peptides provides a new perspective for exploring T1DM pathogenesis and clinical treatments. Furthermore, the detail mechanism of these endogenous peptides to regulate pancreatic $\beta$ cells needs further study.

\section{Data Availability}

The data used to support the findings of this study are included within the article.

\section{Disclosure}

There are no prior publications or submissions with any overlapping information, including studies and patients.

\section{Conflicts of Interest}

The authors have no conflicts of interest.

\section{Authors' Contributions}

Fan Zhang and Meiyun Zhou wrote the first draft of the manuscript. Fan Zhang, Meiyun Zhou, Shuangshuang Li, Jinghua $\mathrm{Gu}$, Yuanyuan Qian, Sisi He, Li Hong, and Linlin Sun carried out the experiments. Weigang Ji and Xiaohua Zhang designed the research. Fan Zhang and Weigang Ji obtained funding. Additionally, there are no payment was given to anyone to produce the manuscript. Fan Zhang and Meiyun Zhou contributed equally to this work.

\section{Acknowledgments}

This work was funded by a grant from the National Natural Science Foundation of China (No. 81800689) and the project of the Nantong Science and Technology Bureau (JCZ20018).

\section{Supplementary Materials}

Table S1: a total of 106 peptides were differentially expressed in the T1DM mice and control groups (fold change $>1.2, p$ $<0.05$ ), including 43 upregulated peptides and 63 downregulated peptides. (Supplementary Materials)

\section{References}

[1] G. Valli, D. Minnock, G. Tarantino, and R. D. Neville, "Delayed effect of different exercise modalities on glycaemic control in type 1 diabetes mellitus: a systematic review and meta-analysis," Nutrition, Metabolism, and Cardiovascular Diseases, vol. 31, no. 3, pp. 705-716, 2021.

[2] N. Kaur, S. K. Bhadada, R. W. Minz, D. Dayal, and R. Kochhar, "Interplay between type 1 diabetes mellitus and celiac disease: implications in treatment," Digestive Diseases, vol. 36, no. 6, pp. 399-408, 2018.

[3] A. Bhatty, S. Baig, A. Fawwad, Z. E. Rubab, M. A. Shahid, and N. Waris, "Association of zinc transporter- 8 autoantibody (ZnT8A) with type 1 diabetes mellitus," Cureus., vol. 12, no. 3, article e7263, 2020.

[4] R. Hanas, C. de Beaufort, H. Hoey, and B. Anderson, "Insulin delivery by injection in children and adolescents with diabetes," Pediatric Diabetes, vol. 12, no. 5, pp. 518-526, 2011.

[5] H. Yu, S. Yu, H. Qiu et al., "Nitric oxide-generating compound and bio-clickable peptide mimic for synergistically tailoring 
surface anti-thrombogenic and anti-microbial dual- functions," Bioactive Materials, vol. 6, no. 6, pp. 1618-1627, 2021.

[6] B. Jones, S. R. Bloom, T. Buenaventura, A. Tomas, and G. A. Rutter, "Control of insulin secretion by GLP-1," Peptides, vol. 100, pp. 75-84, 2018.

[7] J. Sun, L. Furio, R. Mecheri et al., "Pancreatic $\beta$-cells limit autoimmune diabetes via an immunoregulatory antimicrobial peptide expressed under the influence of the gut microbiota," Immunity, vol. 43, no. 2, pp. 304-317, 2015.

[8] J. Sun, M. Xu, H. Ortsäter et al., "Cathelicidins positively regulate pancreatic $\beta$-cell functions," The FASEB Journal, vol. 30, no. 2, pp. 884-894, 2016.

[9] M. Alghrably, I. Czaban, Ł. Jaremko, and M. Jaremko, "Interaction of amylin species with transition metals and membranes," Journal of Inorganic Biochemistry, vol. 191, pp. 6976, 2019.

[10] K. H. Jeong and B. K. Yoo, "The efficacy and safety of liraglutide," International Journal of Clinical Pharmacy, vol. 33, no. 5, pp. 740-749, 2011.

[11] M. Wu, W. Chen, S. Zhang et al., "Rotenone protects against $\beta$-cell apoptosis and attenuates type 1 diabetes mellitus," Apoptosis, vol. 24, no. 11-12, pp. 879-891, 2019.

[12] T. Tomita, "Apoptosis of pancreatic $\beta$-cells in type 1 diabetes," Bosnian Journal of Basic Medical Sciences, vol. 17, no. 3, pp. 183-193, 2017.

[13] S. Nojehdehi, S. Soudi, A. Hesampour, S. Rasouli, M. Soleimani, and S. M. Hashemi, "Immunomodulatory effects of mesenchymal stem cell-derived exosomes on experimental type-1 autoimmune diabetes," Journal of Cellular Biochemistry, vol. 119, no. 11, pp. 9433-9443, 2018.

[14] J. L. Lau and M. K. Dunn, "Therapeutic peptides: historical perspectives, current development trends, and future directions," Bioorganic \& Medicinal Chemistry, vol. 26, no. 10, pp. 2700-2707, 2018.

[15] I. W. Hamley, "Small bioactive peptides for biomaterials design and therapeutics," Chemical Reviews, vol. 117, no. 24, pp. 14015-14041, 2017.

[16] H. Honka and M. Salehi, "Postprandial hypoglycemia after gastric bypass surgery: from pathogenesis to diagnosis and treatment," Current Opinion in Clinical Nutrition and Metabolic Care, vol. 22, no. 4, pp. 295-302, 2019.

[17] X. Shi, S. Chacko, F. Li et al., "Acute activation of GLP-1expressing neurons promotes glucose homeostasis and insulin sensitivity," Molecular Metabolism, vol. 6, no. 11, pp. 13501359, 2017.

[18] F. H. El-Khatib, J. Jiang, and E. R. Damiano, “Adaptive closedloop control provides blood-glucose regulation using dual subcutaneous insulin and glucagon infusion in diabetic swine," Journal of Diabetes Science and Technology, vol. 1, no. 2, pp. 181-192, 2007.

[19] F. S. Malik and C. E. Taplin, "Insulin therapy in children and adolescents with type 1 diabetes," Paediatric Drugs, vol. 16, no. 2, pp. 141-150, 2014.

[20] I. K. Hals, "Treatment of latent autoimmune diabetes in adults: what is best?," Current Diabetes Reviews, vol. 15, no. 3, pp. 188-193, 2019.

[21] A. Y. Y. Cheng, D. K. Patel, T. S. Reid, and K. Wyne, "Differentiating basal insulin preparations: understanding how they work explains why they are different," Advances in Therapy, vol. 36, no. 5, pp. 1018-1030, 2019.
[22] L. Thielen and A. Shalev, "Diabetes pathogenic mechanisms and potential new therapies based upon a novel target called TXNIP," Current Opinion in Endocrinology, Diabetes, and Obesity, vol. 25, no. 2, pp. 75-80, 2018.

[23] M. Cnop, S. Toivonen, M. Igoillo-Esteve, and P. Salpea, "Endoplasmic reticulum stress and eIF $2 \alpha$ phosphorylation: the Achilles heel of pancreatic $\beta$ cells," Molecular Metabolism, vol. 6, no. 9, pp. 1024-1039, 2017.

[24] L. Demirtas, A. Guclu, F. M. Erdur et al., "Apoptosis, autophagy \& endoplasmic reticulum stress in diabetes mellitus," The Indian Journal of Medical Research, vol. 144, no. 4, pp. 515524, 2016.

[25] M. Liu, M. A. Weiss, A. Arunagiri et al., "Biosynthesis, structure, and folding of the insulin precursor protein," Diabetes, Obesity and Metabolism, vol. 20, Suppl 2, pp. 28-50, 2018.

[26] S. Ding, S. Xu, Y. Ma, G. Liu, H. Jang, and J. Fang, "Modulatory mechanisms of the NLRP3 inflammasomes in diabetes," Biomolecules, vol. 9, no. 12, p. 850, 2019.

[27] Z. H. Cao, Z. Wu, C. Hu, M. Zhang, W. Z. Wang, and X. B. Hu, "Endoplasmic reticulum stress and destruction of pancreatic $\beta$ cells in type 1 diabetes," Chinese Medical Journal, vol. 133, no. 1 , pp. $68-73,2020$. 